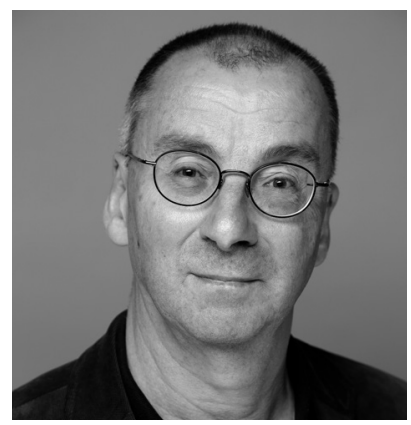

\title{
Kooperation unabdinglich
}

Die Gesundheitswirtschaft gilt der Bundesregierung als »volkswirtschaftlicher Wachstumstreiber «*. In den Befragungen des Branchenreports Gesundheitswirtschaft des Deutschen Industrie- und Handelskammertags vom Herbst 2013 liegen die Geschäftserwartungen der Unternehmen der Gesundheitswirtschaft seit Jahren oberhalb der Prognosen der Gesamtwirtschaft. Im Gesundheitswesen arbeiten derzeit 5,2 Millionen Menschen. Damit ist heute etwa jeder achte Erwerbstätige in dieser Branche tätig. Die Dynamik dieses »Jobmotors Gesundheitswirtschaft « zeigt sich in bemerkenswerten Beschäftigungszuwächsen: Seit dem Jahr 2000 hat die Zahl der Beschäftigten im Gesundheitswesen um über eine Million zugenommen. Damit gehört die Gesundheitswirtschaft wie die Sozialwirtschaft zu den Wachstumssektoren in Deutschland. Beide Arbeitsfelder stehen sich jedoch nach wie vor eher distanziert gegenüber. Neben handfesten gesetzlichen Gründen und unterschiedlichen Finanzierungsregelungen mag dies auch an der Mentalität der Beteiligten liegen. Und doch können beide Sektoren durchaus voneinander profitieren. So sind im Unterschied zur Gesundheitswirtschaft die Leistungsanbieter aus der Sozialwirtschaft vielfach fachlich, verbandlich und räumlich tief gestaffelt. Auch die sozialräumliche Verbundenheit vieler Dienste und Einrichtungen der Sozialen Arbeit kann Ressourcen insbesondere im regionalen und persönlichen Umfeld des Patienten erschließen, von denen Ärzte und Krankenhäuser nicht einmal wissen, dass sie existieren. Zudem entstehen, wie Helmut Hildebrandt und Andreas Brandhorst in ihrem Beitrag in diesem Heft ausführen, durch die Veränderung der Morbidität neue Versorgungsaufgaben, die weniger auf schnelle Heilung ausgerichtet sind und dafür auf Prävention und Rehabilitation, auf palliative Hilfen und auf die Versorgung von an Demenz Erkrankten setzen. Diese Aufgaben erfordern geradezu die Zusammenarbeit zwischen Gesundheitswirtschaft und Sozialwirtschaft.

Gerhard Pfannendörfer, Chefredaktion pfannendoerfer@nomos.de

* Auf der Webseite des Bundesgesundheitsministeriums finden sich zahlreiche Studien und Statistiken zur Bedeutung der Gesundheitswirtschaft in Deutschland, beispielsweise eine Seite mit einem guten Überblick zum Einstieg in das Thema: www.bmg.bund.de/gesundheitssystem/gesundheitswirtschaft/ gesundheitswirtschaft-im-ueberblick.html. 